\title{
Using High Multipolar Orders to Reconstruct the Sound Velocity in Piezoelectrics from Lattice Dynamics
}

\author{
Miquel Royo ${ }^{1},{ }^{, *}$ Konstanze R. Hahn, ${ }^{1}$ and Massimiliano Stengel ${ }^{1,2, \dagger}$ \\ ${ }^{1}$ Institut de Ciència de Materials de Barcelona, ICMAB-CSIC, Campus UAB, 08193 Bellaterra, Spain \\ ${ }^{2}$ ICREA-Institució Catalana de Recerca i Estudis Avançats, 08010 Barcelona, Spain
}

(Received 19 April 2020; accepted 19 October 2020; published 20 November 2020)

\begin{abstract}
Information over the phonon band structure is crucial to predicting many thermodynamic properties of materials, such as thermal transport coefficients. Highly accurate phonon dispersion curves can be, in principle, calculated in the framework of density-functional perturbation theory. However, well-established techniques can run into trouble (or even catastrophically fail) in the case of piezoelectric materials, where the acoustic branches hardly reproduce the physically correct sound velocity. Here we identify the culprit in the higher-order multipolar interactions between atoms and demonstrate an effective procedure that fixes the aforementioned issue. Our strategy drastically improves the predictive power of perturbative latticedynamical calculations in piezoelectric crystals and is directly implementable for high-throughput generation of materials databases.
\end{abstract}

DOI: 10.1103/PhysRevLett.125.217602

The distribution of vibrational frequencies as a function of crystal momentum, known as the phonon band structure, is a key physical property of crystals. Its accurate knowledge is central to predicting several technologically important functionalities, such as thermal expansion [1] and thermoelectric [2-6] coefficients, specific heat $[7,8]$, electron-phonon scattering [9], etc. In many cases, the lowenergy part of the phonon spectrum, consisting of acoustic waves, dominates the aforementioned properties at low to intermediate temperatures. Therefore, for making quantitatively accurate predictions, it is important that the dispersion of the corresponding phonon branches matches the correct sound velocity in a given material.

Density-functional perturbation theory (DFPT) [10-13] has become the state-of-the-art method to calculate the phonon spectrum of crystalline solids from first principles. It allows one to calculate the dynamical matrix, at a computational cost that does not depend on the wave vector $\mathbf{q}$, via the second derivatives of the energy with respect to atomic displacements; subsequent diagonalization yields then the relevant phonon frequencies. Such a procedure could, in principle, be repeated on an arbitrarily dense mesh of $\mathbf{q}$ vectors to integrate the desired thermodynamic function over the full Brillouin zone. This, however, is often impractical; typically, the explicit calculation of the dynamical matrix is carried out on a relatively coarse q mesh only and later Fourier interpolated to a finer grid for thermodynamic integration.

To ensure an accurate interpolation in polar materials, it is crucial to separate the interatomic force constants (IFCs) into a long-ranged dipole-dipole $(D D)$ interaction, which decays as the inverse third power of the interatomic distance $d$, and a "short-ranged" (SR) part, which is simply defined as the remainder. The $D D$ part can be exactly written in terms of two basic ingredients, the Born effective charge tensor $\mathbf{Z}^{*}$ and the macroscopic dielectric tensor $\boldsymbol{\epsilon}_{\infty}$ [13-16]; both can be routinely calculated nowadays for an arbitrary insulator by means of publicly available simulation packages. The SR part, in turn, is assumed to decay sufficiently fast $(D D$ terms indeed constitute the leading contribution at large distances) as a function of $d$ that its Fourier interpolation is efficient and accurate for most purposes.

This procedure yields excellent results in the vast majority of practical cases. The main physical consequence of the $D D$ interactions, namely the frequency splitting between transverse and longitudinal optical phonons at $\Gamma$ [17], is exactly reproduced by construction. Other features of the phonon spectrum typically show optimal convergence even by using relatively coarse q-point meshes [13]. Nevertheless, a number of cases have been reported over the years where unphysical features appear in the interpolated band structures, notably regarding the low-energy bands near the zone center. For example, Refs. [18,19] studied the lattice-dynamical properties of $\mathrm{SiO}_{2}$ across the phase transition from stishovite to $\mathrm{CaCl}_{2}$ structure, finding spurious imaginary acoustic modes in a broad range of pressures around the critical value. Similar imaginary modes can be appreciated in Ref. [20] for $\alpha$-quartz $\mathrm{GeO}_{2}$ and in numerous phonon band structures of piezoelectrics accessible through material databases (see, e.g., $\mathrm{KNbO}_{3}, \mathrm{PNO}, \mathrm{BeSO}_{4}, \mathrm{PdF}_{4}, \mathrm{BPO}_{4}$, or $\mathrm{GaPO}_{4}$ in Ref. [21]). While the aforementioned artifacts were initially ascribed to numerical issues [18] (i.e., to a lack of convergence with respect to the relevant computational parameters), later studies leaned toward a systematic error 
of the Fourier interpolation scheme [19]. The nature of this error, however, has not been clarified yet.

Here we present a rigorous derivation of the long-wave limit in acoustic waves, by generalizing the classic Born and Huang arguments [22] to the case of polar and piezoelectric crystals. In particular, we establish a formal link between the effect of piezoelectrically induced longitudinal fields in elastic waves and nonanalytic contributions to the dynamical matrix beyond the dipole-dipole level. Based on this result, we argue that generalizing the existing theory to the treatment of higherorder multipolar terms (dipole-quadrupole, quadrupolequadrupole, dipole-octupole, and dielectric dispersion effects) is essential to reproducing the correct sound velocity in the phonon dispersion curves. To prove this point, we develop an improved scheme for the Fourier interpolation of phonon bands in insulators, where the aforementioned long-range forces are explicitly treated next to the usual dipole-dipole interactions. By using ferroelectric $\mathrm{BaTiO}_{3}$ as a test case, we demonstrate an extremely rapid convergence of the acoustic branches to the physically correct sound velocity, while spurious imaginary modes are present in the dispersion calculated by ordinary means. Further tests on other problematic cases from the Materials Project database $[23,24]\left(\mathrm{KNbO}_{3}, \mathrm{PNO}, \mathrm{BPO}_{4}\right.$, and $\mathrm{GaPO}_{4}$ ) yield similar results (see Supplemental Material [25]). These unstable modes, which are an artefact of the established Fourier interpolation scheme, persist even in the limit of dense meshes and would thwart any attempt at computing thermodynamic integrals based on such data. Remarkably, our new scheme yields well-behaved (i.e., real) frequencies even in the coarsest $2 \times 2 \times 2$ meshes that we have tested.

The treatment of the acoustic phonons starting from microscopic lattice dynamics occupies an extensive portion of Born and Huang's book [22] and has been revised and extended very recently in the context of flexoelectricity $[38,39]$. We shall start with reviewing the results that are most relevant for the present context. The basic ingredient is the dynamical matrix $(\Phi)$ at some wave vector $\mathbf{q}$, defined as the second derivative of the total energy $(E)$ with respect to two monochromatic perturbations,

$$
\Phi_{\kappa \alpha, \kappa^{\prime} \beta}^{\mathbf{q}}=\frac{\partial^{2} E}{\partial u_{\kappa \alpha}^{\mathbf{q} *} \partial u_{\kappa^{\prime} \beta}^{\mathbf{q}}}, u_{\kappa \alpha}^{l}=u_{\kappa \alpha}^{\mathbf{q}} e^{i \mathbf{q} \cdot \mathbf{R}_{l \kappa}}
$$

Here $\kappa$ and $\kappa^{\prime}$ are sublattice indices, $l$ is a cell index, the real-space vectors $\mathbf{R}_{l \kappa}=\mathbf{R}_{l}+\boldsymbol{\tau}_{\kappa}$ span the crystal lattice, and $\alpha \beta$ are Cartesian directions. Then, the acoustic eigenmodes and velocities can be derived $[22,38,39]$ by performing a perturbative expansion in $\mathbf{q}$ of the lattice-dynamical problem

$$
\sum_{\kappa^{\prime} \beta} \Phi_{\kappa \alpha \kappa^{\prime} \beta}^{\mathbf{q}} u_{\kappa^{\prime} \beta}^{\mathbf{q}}=\omega^{2} m_{\kappa} u_{\kappa \alpha}^{\mathbf{q}}
$$

where $m_{\kappa}$ are atomic masses, $\omega$ is the frequency, and $u_{\kappa \alpha}^{\mathrm{q}}$ are the mode eigenvectors. Following Ref. [39], we shall write $\mathbf{q}=q \hat{\mathbf{q}}$ and take the perturbation expansion in the modulus of the wave vector $q$, while keeping the direction $\hat{\mathbf{q}}$ fixed. The dynamical matrix at small $q$ then reads as

$$
\Phi^{\mathbf{q}}=\Phi^{(0, \hat{\mathbf{q}})}-i q \Phi^{(1, \hat{\mathbf{q}})}-\frac{q^{2}}{2} \Phi^{(2, \hat{\mathbf{q}})}+\cdots
$$

Equation (2) in turn becomes, at second order in $q$,

$$
\sum_{\beta}\left(K_{\alpha \beta}^{\hat{\mathbf{q}}}-M v^{2} \delta_{\alpha \beta}\right) u_{\beta}=0,
$$

where $v$ is the sound velocity, $M$ is the total mass of the cell, $\mathbf{u}$ is the polarization of the phonon branch, and $\mathbf{K}$ is defined as

$$
\begin{aligned}
K^{\hat{\mathbf{q}}} & =-\frac{1}{2} \Phi^{(2, \hat{\mathbf{q}})}+\Phi^{(1, \hat{\mathbf{q}})} \cdot \tilde{\Phi}^{(0, \hat{\mathbf{q}})} \cdot \Phi^{(1, \hat{\mathbf{q}})}, \\
K_{\alpha \beta}^{\hat{\mathbf{q}}} & =\sum_{\kappa \kappa^{\prime}}\left\langle\kappa \alpha\left|K^{\hat{\mathbf{q}}}\right| \kappa^{\prime} \beta\right\rangle .
\end{aligned}
$$

$\tilde{\Phi}^{(0, \hat{\mathbf{q}})}$ denotes the pseudoinverse of the zone-center dynamical matrix; open-circuit electrical boundary conditions are assumed along $\hat{\mathbf{q}}$ for all quantities in Eq. (5).

After careful considerations of the nonanalytic behavior of $\Phi^{\mathbf{q}}$ near the zone center, we find [25]

$$
K_{\alpha \beta}^{\hat{\mathbf{q}}}=\Omega \sum_{\gamma \delta} \hat{q}_{\gamma} C_{\alpha \gamma \beta \delta}^{\hat{\mathbf{q}}} \hat{q}_{\delta} .
$$

where $\mathbf{C}^{\hat{\mathbf{q}}}$ is the elastic tensor in "mixed electrical boundary conditions" [40,41] (open circuit is imposed along $\hat{\mathbf{q}}$ ),

$$
C_{\alpha \gamma \beta \delta}^{\hat{\mathbf{q}}}=C_{\alpha \gamma \beta \delta}+4 \pi \frac{(\hat{\mathbf{q}} \cdot \mathbf{e})_{\alpha \gamma}(\hat{\mathbf{q}} \cdot \mathbf{e})_{\beta \delta}}{\hat{\mathbf{q}} \cdot \boldsymbol{\epsilon} \cdot \hat{\mathbf{q}}} .
$$

Here $\mathbf{C}$ is the elastic tensor calculated in short circuit, and the second term on the rhs embodies the directiondependent macroscopic electric field contribution via the piezoelectric (e) and dielectric ( $\boldsymbol{\epsilon})$ tensors. (All three tensors are defined in the static limit, i.e., inclusive of lattice-mediated contributions.) Thus, Eq. (4) exactly reduces to the macroscopic Christoffel equation $[42,43]$ for sound waves in a crystalline insulator of arbitrary symmetry.

The above derivations establish the formal connection between macroscopic elasticity and microscopic lattice dynamics by generalizing the classic arguments of Born and Huang [22] to an arbitrary crystal, including polar and piezoelectric insulators. The link between long-wavelength phonons and Eq. (8) is provided by Martin's formula [44] for the macroscopic piezoelectric tensor, where the latter is written in terms of dynamical dipoles and quadrupoles 
associated with atomic displacements. Thus, from these derivations we have learned a crucially important fact: to reproduce the correct sound velocity in a phonon spectrum calculation of a piezoelectric material, higher-order multipolar contributions (e.g., involving dynamical quadrupoles [45]) to the IFCs play a key role.

To see the implications of this statement in the context of first-principles lattice dynamics, we shall recap the state-ofthe-art method for the Fourier interpolation of the phonon bands in insulating crystals. $\Phi^{\mathbf{q}}$ is typically calculated within density-functional perturbation theory on a "coarse" mesh of $\mathbf{q}_{i}$ points spanning the Brillouin zone and later interpolated on a much finer mesh for computing various thermodynamic quantities. To this end, one first defines a "long-range" (LR) dipole-dipole contribution in terms of the Born effective charge and dielectric tensors and subtracts it from the calculated $\Phi\left(\mathbf{q}_{i}\right)$,

$$
\Phi^{\mathrm{SR}}\left(\mathbf{q}_{i}\right)=\Phi\left(\mathbf{q}_{i}\right)-\Phi^{\mathrm{LR}}\left(\mathbf{q}_{i}\right) .
$$

Next, the remainder SR part is backward Fourier transformed to obtain the real-space IFCs on a supercell $\mathcal{S}$ that is dual to the coarse $\mathbf{q}$ mesh. ( $\mathcal{S}$ is assumed to be a polyhedron centered at the origin; the IFCs are conveniently truncated at the boundaries according to the interatomic distance.) Finally, the dynamical matrix at an arbitrary point $\mathbf{q}$ is reconstructed by adding back the dipole-dipole term to the Fourier-interpolated (IN) short-range part,

$$
\Phi^{\mathrm{tot}}(\mathbf{q})=\Phi^{\mathrm{IN}}(\mathbf{q})+\Phi^{\mathrm{LR}}(\mathbf{q})
$$

where the latter are defined as

$$
\Phi_{\kappa \alpha, \kappa^{\prime} \beta}^{\mathrm{IN}, \mathbf{q}}=\sum_{l: \mathbf{d}_{\kappa k^{\prime}}^{l} \in \mathcal{S}} \Phi_{\kappa \alpha, \kappa^{\prime} \beta}^{\mathrm{SR}, l} e^{-i \mathbf{q} \cdot \mathbf{d}_{\kappa k^{\prime}}^{l}} .
$$

$\left(\mathbf{d}_{\kappa \kappa^{\prime}}^{l}=\mathbf{R}_{l}+\boldsymbol{\tau}_{\kappa^{\prime}}-\boldsymbol{\tau}_{\kappa}\right.$ is the real-space vector connecting atoms $0 \kappa$ and $l \kappa^{\prime}$.)

We can now connect to Eq. (4) by expanding the interpolated dynamical matrix in powers of $q$, similar to Eq. (3). We shall specifically focus on $\Phi^{\mathrm{IN}, \mathbf{q}}$, since $\Phi^{\mathrm{LR}}$ is defined by analytical formulas and therefore trivial to deal with in this context. We find that the $n$th expansion term is trivially given by the real-space moments of the shortrange IFC,

$$
\Phi_{\kappa \alpha, \kappa^{\prime} \beta}^{\mathrm{IN}-(n, \hat{\mathbf{q}})}=\sum_{l: \mathbf{d}_{\kappa k^{\prime}}^{l} \in \mathcal{S}} \Phi_{\kappa \alpha, \kappa^{\prime} \beta}^{\mathrm{SR}, l}\left(\mathbf{d}_{\kappa \kappa^{\prime}}^{l} \cdot \hat{\mathbf{q}}\right)^{n} .
$$

The validity of the interpolation procedure for the longwavelength acoustic waves therefore rests on the accuracy of Eq. (12) and, in particular, on whether the lattice sums up to $n=2$ are well defined. [Equation (4) contains $q$ derivatives of the dynamical matrix up to second order.]

A sufficient condition for the $n$th moment to converge is that the SR interatomic force constants decay faster than
$1 / d^{3+n}$, since the sum must be performed over the threedimensional volume of $\mathcal{S}$. Thus, in our case we must require that $\Phi_{\kappa \alpha, \kappa^{\prime} \beta}^{\mathrm{SR}, l}$ decay faster than $1 / d^{5}$. Within the standard interpolation method, the decay rate, however, is only guaranteed to be faster than $1 / d^{3}$, since the dipoledipole interactions are subtracted out from the IFC calculated from first principles, but higher-order multipolar interactions (e.g., dipole-quadrupole, decaying as $\sim 1 / d^{4}$ ) are generally present. As a consequence, the lattice sums for $n=1,2$ in Eq. (12) are, in principle, only conditionally convergent. This means that the sound velocity that one extracts from the interpolated phonon dispersion curves may depend on the details of how the IFCs are truncated at the boundary, i.e., on the shape of the supercell $\mathcal{S}$ that one uses in practice. Note that this statement holds even in the limit of a very large supercell size, so in severe cases this issue may be difficult or impossible to solve by simply increasing the density of the coarse $\mathbf{q}$ mesh.

To solve this issue, we shall rewrite the long-range contribution of Eqs. (9) and (10) by incorporating enough terms to reproduce the nonanalyticities of $\Phi(\mathbf{q})$ up to $O\left(q^{2}\right)$,

$$
\begin{aligned}
\Phi^{\mathrm{LR}}(\mathbf{q})= & \Phi^{D D}(\mathbf{q})+\Phi^{D Q}(\mathbf{q}) \\
& +\Phi^{D O}(\mathbf{q})+\Phi^{Q Q}(\mathbf{q})+\Phi^{D \epsilon D}(\mathbf{q}),
\end{aligned}
$$

Here $D, Q$, and $O$ stand for dipole, quadrupole, and octupole, respectively. The last term on the rhs $(D \epsilon D)$ is a dipole-dipole term mediated by the dielectric dispersion (see Supplemental Material [25]). The modification of the LR part redefines the short-range IFCs as well, which are now guaranteed to decay as $1 / d^{6}$ or faster (all interactions up to $1 / d^{5}$ have been removed), as required by Eq. (5). Interestingly, in addition to the $D Q$ and $Q Q$ interactions (whose importance for piezoelectric crystals was formally demonstrated in the earlier paragraphs), we have two additional $O\left(q^{2}\right)$ terms here, $D O$ and $D \epsilon D$, whose significance may be at first sight unclear. If $\mathcal{S}$ were infinitely large, neither interaction should have an impact on the sound velocity-the corresponding electrostatic contributions to the acoustic branches would vanish because of the acoustic sum rule (ASR) [25]. Yet, at finite $\mathcal{S}$ size, the abrupt truncation of the IFCs at the boundary might spoil the ASR at the level of the $D O$ and $D \epsilon D$ terms, resulting in a slow convergence of the interpolated sound velocity with q mesh resolution. We shall see shortly, by using rhombohedral $\mathrm{BaTiO}_{3}$ as a test case, an excellent practical demonstration of these arguments: while inclusion of dipole-quadrupole terms produces the most dramatic effects, $D O$ and $D \epsilon D$ terms further improve the convergence rate of the interpolated sound velocities and rather substantially so.

We have implemented the above procedure [25] to calculate the higher-order long-range interactions into the ANADDB postprocessing program, which is part of 


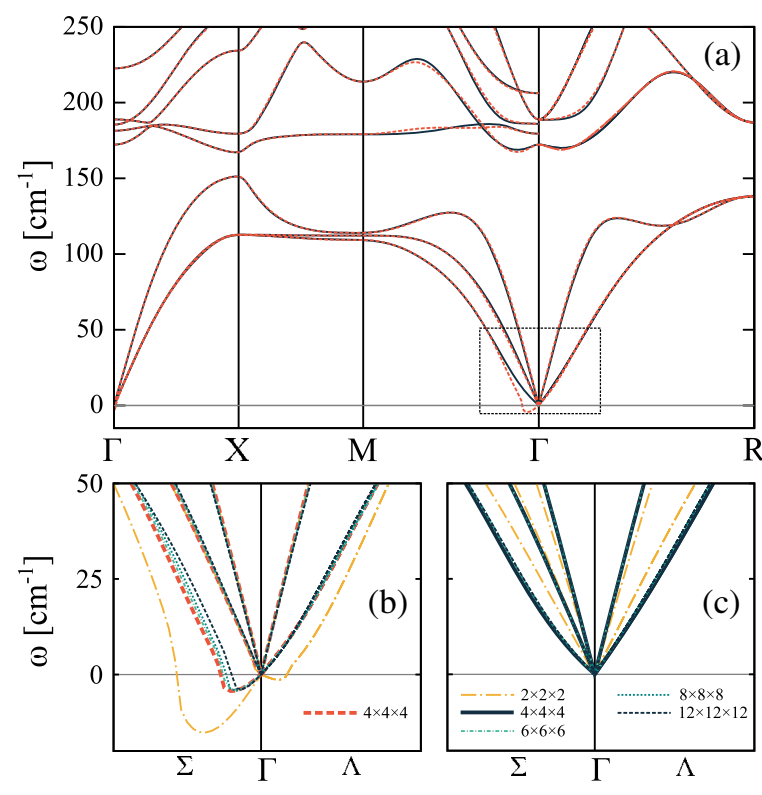

FIG. 1. (a) Phonon dispersion of $\mathrm{BaTiO}_{3}$ calculated using the standard $D D$-based procedure (red dashed lines) and our higherorder interpolation scheme, based on Eq. (13) (black solid lines); a $4 \times 4 \times 4$ q-points mesh was used in both cases. The bottom panels show an enlargement of the acoustic bands over the region marked by a dashed rectangle in (a). The additional (thinner) curves were obtained using different q-point meshes [see the legend of (c)], either with the standard procedure (b) or Eq. (13) (c).

the ABINIT suite. (The $\mathrm{v} 9$ version of ABINIT with this new functionality has just been released for public use [46].) The phonon band dispersion evaluated along the path $\Gamma-X-M-\Gamma-R$ and calculated with a $4 \times 4 \times 4$ q-points mesh is represented in Fig. 1. Red dashed lines show the results obtained following the standard procedure [16,47], in which $\Phi^{\mathrm{LR}}(\mathbf{q})$ exclusively includes dipole-dipole interactions. The optical bands resulting from this calculation show no visible anomalies. However, a sizeable portion of one of the transverse acoustic bands dips into imaginary frequencies at the long-wave limit of the $\Gamma-M$ (corresponding to [110]) segment. Such anomaly does not disappear by increasing the density of the coarse $\mathbf{q}$ meshspurious imaginary modes persist along [110] up to the highest-density mesh we could realistically afford $(12 \times 12 \times 12)$, as illustrated in Fig. 1(b). To confirm that this artifact is indeed related to the Fourier interpolation scheme, we have performed explicit DFPT calculations of the phonon frequencies at selected (small) $\mathbf{q}$ values along [110], always obtaining real frequencies. Also, this is certainly not the signature of a physical ferroelastic instability of the crystal, since the $\mathrm{BaTiO}_{3}$ cell has been carefully relaxed to its well-known low-temperature rhombohedral structure.

The black solid lines of Fig. 1(a) were calculated based on our higher-order multipolar interpolation scheme of

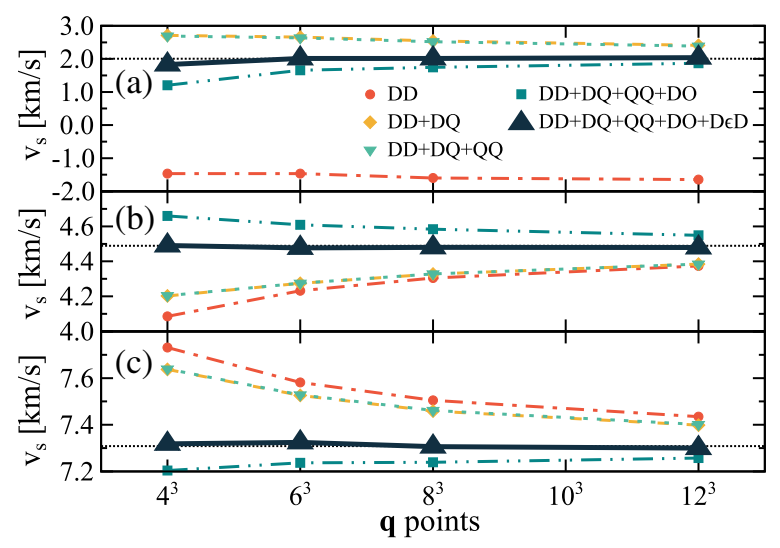

FIG. 2. Velocity of sound of the three acoustic branches along the [110] direction as a function of the $\mathbf{q}$-point mesh resolution. Dotted horizontal lines indicate the reference value of the sound velocity, obtained from macroscopic elasticity via Eqs. (4), (7), and (8). Different symbols (lines are a guide to the eye) show the velocities as obtained by considering an increasing number of multipolar interactions in Eq. (13).

Eq. (13). Remarkably, imaginary frequencies disappear even for the coarsest $2 \times 2 \times 2 \mathbf{q}$ grid [see Fig. 1(c)], and the dispersion of all acoustic branches shows optimal convergence already for a $4 \times 4 \times 4$ mesh. Our revised scheme seems to improve the description of some optical branches as well, most notably of the lowest-energy (ferroelectric) mode along the $\Gamma-M$ and the $\Gamma-R$ directions, although the corrections appear to be comparatively less important.

To make the above statements more quantitative, we extract the propagation velocities of the three acoustic waves from the dispersion curves of Fig. 1 and compare them with the macroscopic results, based on Eqs. (4), (7), and (8) (values are reported in Table SV of the Supplemental Material [25]). The velocities along the [110] direction are shown in Fig. 2 (data along [100] and [111] can be found in Fig. S1 of the Supplemental Material [25]) as a function of the $\mathbf{q}$-point mesh resolution. In order to illustrate the effect of each individual multipolar term, we have recalculated the sound velocity several times by progressively incorporating an increasing number of the terms on the rhs of Eq. (13). Incorporation of the $D Q$ interactions drastically improves the accuracy of the estimated velocity of sound, completely removing the spurious imaginary modes along all directions, as we said. However, only treating electrostatic terms up to $O\left(q^{1}\right)$ clearly does not guarantee accurate results in this case. Indeed, such an approximation leads to an error of the order of $10 \%-20 \%$ in the velocities that decays only slowly as a function of the q-grid resolution.

Including the $O\left(q^{2}\right)$ electrostatic interactions produces a further remarkable improvement in the accuracy: the dispersion of the acoustic branches is essentially converged to the correct sound velocity already at a $\mathbf{q}$ mesh resolution 


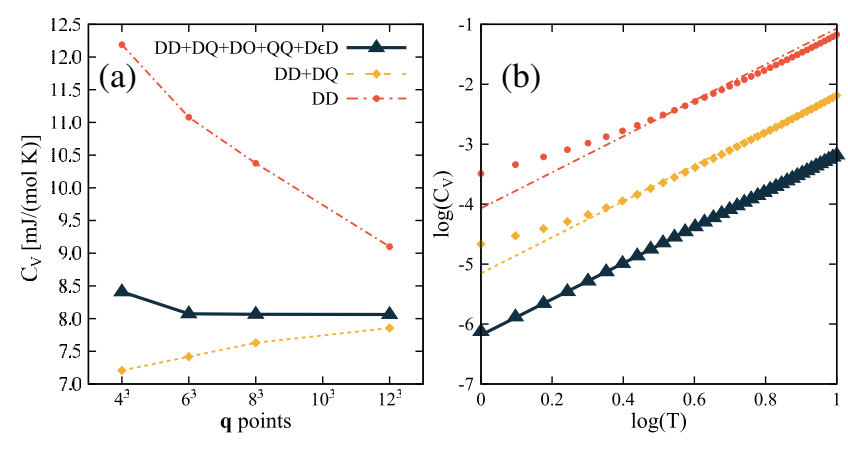

FIG. 3. (a) Specific heat $\left(C_{V}\right)$ computed at $T=5 \mathrm{~K}$ from the phonon energy spectrum as a function of the coarse q-point mesh by using three different interpolation methods (see text). (b) $\log \left(C_{V}\right)$ vs $\log (T)$ data obtained with a $12 \times 12 \times 12$ coarse mesh. Lines show a logarithmic fit to the Debye law, $C_{V} \propto T^{3}$. Vertical labels correspond to the $D D$ data; the other two sets have been shifted by -1 and -2 for clarity. A fine $\mathbf{q}$ mesh of $40 \times 40 \times 40$ is used for the integration in all cases.

of $4 \times 4 \times 4$. This result clearly supports our formal arguments of the previous paragraphs. Interestingly, among the three $O\left(q^{2}\right)$ interactions $Q Q$ have a negligible effect, which is a bit surprising considering that $Q Q$ terms should play an important role in piezoelectrics. This is likely due to the fact that the piezoelectric coefficients in ferroelectric materials such as $\mathrm{BaTiO}_{3}$ are dominated by lattice-mediated contributions, while clamped-ion effects are comparatively negligible. $[Q Q$ interactions microscopically embody the contribution of $\overline{\mathbf{e}}$ to the dynamics of acoustic waves, see Eq. (S22) [25].] Indeed for $\mathrm{BaTiO}_{3}$ we obtain a difference of one order of magnitude between both contributions (see Table SVI of the Supplemental Material [25]). To verify the validity of this hypothesis, we tested our method on a different material, GaP. In zinc blende semiconductors, the lattice-mediated and clamped-ion contributions to the piezoelectric tensor are generally similar in magnitude and opposite in sign (see, e.g., Table SVI [25]), which makes $\mathrm{GaP}$ an excellent counterexample. And indeed, as we show in Fig. S2 [25], $D Q$ and $Q Q$ corrections are nearly equal and opposite, confirming our arguments above.

To assess the impact of our method on the calculation of thermal properties, we have computed the low-temperature specific heat [7] of rhombohedral $\mathrm{BaTiO}_{3}$. In Fig. 3(a) we show the calculated values of $C_{V}(T=5 \mathrm{~K})$ as a function of the $\mathbf{q}$ mesh resolution. Our method, as expected, yields a dramatically improved convergence rate compared to the standard $D D$-based treatment. Note that inclusion of the $D Q$ interactions already reduces the error by approximately one order of magnitude. In Fig. 3(b), we show a log-log plot of $C_{V}(T),(T=0.25-10 \mathrm{~K})$ calculated at fixed mesh resolution of $12 \times 12 \times 12$. By using our higher-order method, the results accurately reproduce the lowtemperature limit $\left(\sim T^{3}\right)$ of Debye's law [48]; the fitted Debye temperature, $T_{D}=530 \mathrm{~K}$, is in good agreement with existing experimental and theoretical values [49].
Conversely, the standard $D D$-based approach shows important deviations, pointing to a qualitative, rather than quantitative, misrepresentation of the low-energy part of the phonon spectrum. Interestingly, $D Q$ terms alone are clearly unable to correct this flaw, indicating that the absence of spurious imaginary branches is not per se sufficient to guarantee that the relevant physical properties are well represented.

To summarize, by including higher-order multipolar interactions in the determination of interatomic force constants, we were able to eliminate spurious artifacts in the phonon dispersion spectrum of $\mathrm{BaTiO}_{3}$ and obtain a remarkably accurate description of the acoustic branches even at small $\mathbf{q}$ mesh resolutions. Unphysical acoustic imaginary modes are not exclusive of the $\mathrm{BaTiO}_{3}$ system studied here. Indeed, materials databases, such as Refs. [21,50], are riddled with piezoelectrics developing these kinds of artifacts. The implementation shown here can be readily applied for improving the high-throughput generation of phonon band structures to be included in these and other databases.

We acknowledge the support of Ministerio de Economia, Industria y Competitividad (MINECO-Spain), through Grants No. MAT2016-77100-C2-2-P and No. SEV-20150496, and of Generalitat de Catalunya (Grant No. 2017 SGR1506). This project has received funding from the European Research Council (ERC) under the European Union's Horizon 2020 Research and Innovation Program (Grant Agreement No. 724529). Part of the calculations were performed at the Supercomputing Center of Galicia (CESGA).

*mroyo@icmab.es

†mstengel@icmab.es

[1] A. Fleszar and X. Gonze, First-Principles Thermodynamical Properties of Semiconductors, Phys. Rev. Lett. 64, 2961 (1990).

[2] D. A. Broido, M. Malorny, G. Birner, N. Mingo, and D. A. Stewart, Intrinsic lattice thermal conductivity of semiconductors from first principles, Appl. Phys. Lett. 91, 231922 (2007).

[3] A. Ward, D. A. Broido, D. A. Stewart, and G. Deinzer, $A b$ initio theory of the lattice thermal conductivity in diamond, Phys. Rev. B 80, 125203 (2009).

[4] A. Ward and D. A. Broido, Intrinsic phonon relaxation times from first-principles studies of the thermal conductivities of Si and Ge, Phys. Rev. B 81, 085205 (2010).

[5] G. Fugallo, M. Lazzeri, L. Paulatto, and F. Mauri, Ab initio variational approach for evaluating lattice thermal conductivity, Phys. Rev. B 88, 045430 (2013).

[6] W. Li, J. Carrete, N. A. Katcho, and N. Mingo, ShengBTE: A solver of the Boltzmann transport equation for phonons, Comput. Phys. Commun. 185, 1747 (2014).

[7] C. Lee and X. Gonze, Ab initio calculation of the thermodynamic properties and atomic temperature factors of $\mathrm{SiO}_{2}$ $\alpha$-quartz and stishovite, Phys. Rev. B 51, 8610 (1995). 
[8] M. Sanati and S. K. Estreicher, Specific heat and entropy of GaN, J. Phys. Condens. Matter 16, L327 (2004).

[9] F.Giustino, Electron-phonon interactions from first principles, Rev. Mod. Phys. 89, 015003 (2017).

[10] S. Baroni, P. Giannozzi, and A. Testa, Green's-Function Approach to Linear Response in Solids, Phys. Rev. Lett. 58, 1861 (1987).

[11] X. Gonze, Adiabatic density-functional perturbation theory, Phys. Rev. A 52, 1096 (1995).

[12] X. Gonze, Adiabatic density-functional perturbation theory, Phys. Rev. A 54, 4591(E) (1996).

[13] S. Baroni, S. De Gironcoli, A. Dal Corso, and P. Giannozzi, Phonons and related crystal properties from densityfunctional perturbation theory, Rev. Mod. Phys. 73, 515 (2001).

[14] P. Giannozzi, S. de Gironcoli, P. Pavone, and S. Baroni, $A b$ initio calculation of phonon dispersions in semiconductors, Phys. Rev. B 43, 7231 (1991).

[15] X. Gonze, First-principles responses of solids to atomic displacements and homogeneous electric fields: Implementation of a conjugate-gradient algorithm, Phys. Rev. B 55, 10337 (1997).

[16] X. Gonze and . Lee, Dynamical matrices, Born effective charges, dielectric permittivity tensors, and interatomic force constants from density-functional perturbation theory, Phys. Rev. B 55, 10355 (1997).

[17] W. Cochran, Lattice dynamics of sodium, Proc. R. Soc. Ser. A 276, 308 (1963).

[18] A. Togo, F. Oba, and I. Tanaka, First-principles calculations of the ferroelastic transition between rutile-type and $\mathrm{CaCl}_{2}$ type $\mathrm{SiO}_{2}$ at high pressures, Phys. Rev. B 78, 134106 (2008).

[19] H. Aramberri, R. Rurali, and J. Íñiguez, Thermal conductivity changes across a structural phase transition: The case of high-pressure silica, Phys. Rev. B 96, 195201 (2017).

[20] P. Hermet, A. Lignie, G. Fraysse, P. Armand, and P. Papet, Thermodynamic properties of the $\alpha$-quartz-type and rutiletype GeO2 from first-principles calculations, Phys. Chem. Chem. Phys. 15, 15943 (2013).

[21] A. Togo, phonondb@kyoto-u [2015 (accessed, 2020)], http://phonondb.mtl.kyoto-u.ac.jp.

[22] M. Born and K. Huang, in Dynamical Theory of Crystal Lattices (Oxford University Press, Oxford, 1954), p. 420.

[23] A. Jain, S. P. Ong, G. Hautier, W. Chen, W. D. Richards, S. Dacek, S. Cholia, D. Gunter, D. Skinner, G. Ceder, and K. A. Persson, The materials project: A materials genome approach to accelerating materials innovation, APL Mater. 1, 011002 (2013).

[24] G. Petretto, S. Dwaraknath, H. P. C. Miranda, D. Winston, M. Giantomassi, M. J. van Setten, X. Gonze, K. A. Persson, G. Hautier, and G.-M. Rignanese, High-throughput densityfunctional perturbation theory phonons for inorganic materials, Sci. Data 5, 180065 (2018).

[25] See Supplemental Material at http://link.aps.org/ supplemental/10.1103/PhysRevLett.125.217602 for more information about computational details and results, which include Refs. [26-37].

[26] W. H. Press, S. A. Teukolsky, W. T. Vetterling, and B. P. Flannery, in Numerical Recipes in Fortran 77: The Art of
Scientific Computing, 2nd ed. (Cambridge University Press, Cambridge, 1996) Vol. 1, Chap. 2, p. 65.

[27] X. Gonze et al., ABINIT: First-principles approach to material and nanosystem properties, Comput. Phys. Commun. 180, 2582 (2009).

[28] X. Gonze et al., Recent developments in the ABINIT software package, Comput. Phys. Commun. 205, 106 (2016).

[29] J. P. Perdew and Y. Wang, Accurate and simple analytic representation of the electron-gas correlation energy, Phys. Rev. B 45, 13244 (1992).

[30] C. E. Dreyer, M. Stengel, and D. Vanderbilt, Current-density implementation for calculating flexoelectric coefficients, Phys. Rev. B 98, 075153 (2018).

[31] A. Schiaffino, C. E. Dreyer, D. Vanderbilt, and M. Stengel, Metric wave approach to flexoelectricity within density functional perturbation theory, Phys. Rev. B 99, 085107 (2019).

[32] D. R. Hamann, X. Wu, K. M. Rabe, and D. Vanderbilt, Metric tensor formulation of strain in density-functional perturbation theory, Phys. Rev. B 71, 035117 (2005).

[33] R. Resta, Towards a Bulk Theory of Flexoelectricity, Phys. Rev. Lett. 105, 127601 (2010).

[34] J. Hong and D. Vanderbilt, First-principles theory of frozen-ion flexoelectricity, Phys. Rev. B 84, 180101(R) (2011).

[35] M. Stengel and D. Vanderbilt, First-principles theory of flexoelectricity, in Flexoelectricity in Solids From Theory to Applications, edited by A. K. Tagantsev and Petr V. Yudin (World Scientific Publishing Co., Singapore, 2016), Chap. 2, pp. 31-110.

[36] D. R. Hamann, Optimized norm-conserving Vanderbilt pseudopotentials, Phys. Rev. B 88, 085117 (2013).

[37] M. J. van Setten, M. Giantomassi, E. Bousquet, M. J. Verstraete, D. R. Hamann, X. Gonze, and G.-M. Rignanese, The PseudoDojo: Training and grading a 85 element optimized norm-conserving pseudopotential table, Comput. Phys. Commun. 226, 39 (2018).

[38] M. Stengel, Flexoelectricity from density-functional perturbation theory, Phys. Rev. B 88, 174106 (2013).

[39] M. Stengel, Unified ab initio formulation of flexoelectricity and strain-gradient elasticity, Phys. Rev. B 93, 245107 (2016).

[40] X. Wu, D. Vanderbilt, and D. R. Hamann, Systematic treatment of displacements, strains, and electric fields in density-functional perturbation theory, Phys. Rev. B 72, 035105 (2005).

[41] J. Hong and D. Vanderbilt, First-principles theory and calculation of flexoelectricity, Phys. Rev. B 88, 174107 (2013).

[42] Z. Li, M. Grimsditch, C. M. Foster, and S.-K. Chan, Dielectric and elastic properties of ferroelectric materials at elevated temperature, J. Phys. Chem. Solids 57, 1433 (1996).

[43] A. N. Abd-alla and N. A. Askar, Calculation of bulk acoustic wave propagation velocities in trigonal piezoelectric smart materials, Appl. Math. Inf. Sci. 8, 1625 (2014).

[44] R. M. Martin, Piezoelectricity, Phys. Rev. B 5, 1607 (1972). 
[45] M. Royo and M. Stengel, First-Principles Theory of Spatial Dispersion: Dynamical Quadrupoles and Flexoelectricity, Phys. Rev. X 9, 021050 (2019).

[46] A. H. Romero et al., ABINIT: Overview and focus on selected capabilities, J. Chem. Phys. 152, 124102 (2020).

[47] X. Gonze, J.-C. Charlier, D. C. Allan, and M. P. Teter, Interatomic force constants from first principles: The case of $\alpha$-quartz, Phys. Rev. B 50, 13035 (1994).
[48] C. Kittel, Introduction to Solid State Physics, 6th ed. (John Wiley \& Sons, Inc., New York, 1986).

[49] S. Sanna, C. Thierfelder, S. Wippermann, T. P. Sinha, and W. G. Schmidt, Barium titanate ground- and excited-state properties from first-principles calculations, Phys. Rev. B 83, 054112 (2011).

[50] H. P. C. Miranda, Phonon website (2015 [accessed 2020]), http://henriquemiranda.github.io/phononwebsite. 